\title{
RETRACTION
}

\section{Kinetic Resistance of Carbon Fullerenes in Aqueous Solution}

\author{
[NANO: Brief Reports and Reviews, Vol. 2, No. 4 (2007), \\ DOI: $10.1142 /$ S1793292007000556] \\ Haijun Shen \\ School of Aeronautics and Astronautics \\ Nanjing University of Aeronautics and Astronautics \\ Nanjing, Jiangsu 210016, P. R. China \\ shj@nuaa.edu.cn
}

Published 2 May 2019

\begin{abstract}
This article has been retracted at the request of the Editors. It is fully identical with another article Kinetic resistance of carbon fullerenes in aqueous solution which was published in Computers and Applied Chemistry Vol. 24 No. 6 (June 2007) 819-822. It is inappropriate and violates the scientific rule of anti-plagiarism. We regret that this was not detected before the article was accepted for publication in the journal.
\end{abstract}

